\title{
Quality of Care among Non-Communicable Diseases (NCDs) Service Delivery in South Asia: A Systematic Rapid Review
}

\author{
Saraban Ether \\ Maternal and Child Health Division, icddr,b \\ K M Saif-Ur-Rahman ( $\square$ su.rahman@icddrb.org ) \\ Health Systems and Population Studies Division, icddr,b
}

\section{Research Article}

Keywords: Quality of Care, NCDs, Service delivery, South Asia, Systematic review

Posted Date: February 8th, 2021

DOI: https://doi.org/10.21203/rs.3.rs-155655/v1

License: (1) (i) This work is licensed under a Creative Commons Attribution 4.0 International License. Read Full License

Version of Record: A version of this preprint was published at Public Health in Practice on November 1st, 2021. See the published version at https://doi.org/10.1016/j.puhip.2021.100180. 


\section{Abstract}

Background: South Asia is facing the challenges of non-communicable diseases (NCDs) which are getting doubled due to the low quality of care (QoC) around NCD services. This systematic rapid review aims to unpack available approaches to establish the quality of care around NCD services and the impact of those approaches to reduce NCD burden in South Asian countries.

Methods: Three electronic databases (Medline, Embase, and the Cochrane Library) were searched. Studies published from 1st January 1990 to 31st December 2020 were included. Studies written in English in the South Asian context following any research design about four major NCDs (cancer, diabetes, cardiovascular disease, chronic respiratory disease), and interventions to achieve QoC were included. Data extraction was done using a pre-specified form. A narrative synthesis was conducted for analyzing the extracted information. This systematic rapid review is registered in PROSPERO (International prospective register of systematic reviews) - CRD42020157401.

Results: Among 829 identified studies 13 were included in the review for in-depth analysis. Most of the studies focused on cancer followed by diabetes and cardiovascular disease. Community and clinic-based screening, NCD care education, NCD specialized corner or hospital, and a follow-up system ensure patient satisfaction, accessibility, early detection, timely referral, and help to reduce disease severity, mortality rate, and incidence of the new disease.

Conclusions: The effective interventions for improving QoC around NCD services can be scaled up in different settings in South Asia to reduce the burden of NCDs.

\section{Background:}

Worldwide non-communicable diseases (NCDs) have become the leading cause of premature death due to the global epidemiological transition. In 2016, worldwide 56.9 million deaths occurred and NCDs attributed 71\% (40.5 million) of that total death. Premature deaths due to NCDs in low- and middle-income countries (LMICs) was accounted for $46 \%$ of 31.5 million deaths ${ }^{1}$.

Unsurprisingly, South Asia is facing an epidemiological transition from communicable diseases to NCDs. The percentage of NCD-related deaths out of the total number of deaths in South Asian countries ranges from 44-84\%. NCD related premature deaths statistics in these countries share a similar trajectory ${ }^{2}$.

People of South Asia is suffering from all four major types of NCDs. Recent cancer statistics showed that 1 million out of 1.3 billion people were diagnosed with cancer annually in India and in 2012 and 600,000 to 700,000 deaths attributed to cancer. However, the International Agency for Research on Cancer (GLOBOCAN) has predicted that this number will double in South Asia in the next 20 years ${ }^{3}$. South Asians faced a quadrupled risk of developing diabetes, compared to other ethnic groups, and the changed lifestyle of people living in this continent make it worse 4,5 . It is estimated that, by 2030 , the total number of people affected by diabetes in India, Pakistan, and Bangladesh will reach 46,14 , and 11.1 million respectively ${ }^{6}$. Out of 17 million NCD related premature deaths in the whole world in $2015,37 \%$ occurred in LMICs due to cardiovascular diseases (CVD) ${ }^{7}$. In 2017, data published by the World Health Organisation (WHO) revealed that CVD accounted for a considerable amount of total deaths in South Asia ${ }^{8}$. Worldwide chronic respiratory disease is the third cause of death ${ }^{9}$ and in LMICs $>90 \%$ of deaths are the reason for Chronic obstructive pulmonary disease (COPD) ${ }^{10}$. Chronic respiratory disease accounted for $3 \%-11 \%$ of total NCD- 
related deaths in South Asia ${ }^{2}$. Due to the huge burden of NCDs in South Asia, the global development community is committed to a global target of reducing premature mortality from NCDs by one-third before $2030{ }^{11}$.

The South Asian Region is densely populated, and half of the total population lives below the poverty line ${ }^{12}$. It was estimated that about 8.6 million deaths were occurring in LMICs, due to the lack of appropriate quality care ${ }^{13}$. As a result, it is crucial to maintain the quality of care (QoC) in NCD services in South Asia. In this review the concept of QoC was adapted from WHO as "health care must be safe, effective, timely, efficient, equitable and people-centred" for achieving QoC in health care services ${ }^{14}$.

There are limited studies where QoC around NCD services were discussed concurrently. A systematic review reported only one study which provided the idea that there was difficulty in identifying scientific articles on major NCDs and approach to ensure QoC ${ }^{15}$. Other reviews described interventions to manage NCDs ${ }^{16}$ and service availability around NCDs but the QoC issue was absent ${ }^{17}$.

No systematic review focusing to ensure QoC for NCD services in South Asia was identified. As a result, this review is essential because of the high NCD burden, and little attention to QoC around NCD services. Thus, this review aims to investigate available interventions to identify QoC around NCD services, and the impact of these interventions to reduce the NCD burden in South Asian countries.

\section{Methods:}

A systematic rapid review was done which followed the recommended rapid review guideline by WHO ${ }^{18}$ and preferred reporting items for systematic review and meta-analysis (PRISMA) guidelines. This systematic rapid review is registered in PROSPERO (International prospective register of systematic reviews) - CRD42020157401.

\section{Study population}

NCD services for all people living in the South Asian region were considered.

\section{Intervention}

Studies discussed the interventions implemented by both public and private ventures related to QoC dimensions such as people-centeredness, accessibility, continuity of care, safety, acceptability, equity, effectiveness, efficiency 14 in the health system were included in this review.

\section{Comparison and type of studies}

No comparison group was required in studies included in this review.

\section{Outcome}

Reduction of mortality, morbidity, the incidence of a disease and health inequalities, increasing life expectancy, patient satisfaction, accessibility and acceptability of health services, shortening patient's waiting time, improving outcomes for disease and efficiency of NCD health services were the outcomes that were considered. 


\section{Study selection, timeline, and language}

This review was not limited to any study design and considered studies published in the English language only. The timeline of this review ranged from 1st January 1990 to 31st December 2020 as there has been a steady increase in NCDs in South Asian countries since $1990{ }^{12}$.

\section{Search strategy}

The comprehensive search strategy was applied to retrieve articles from three databases (Medline, EMBASE, Cochrane database). It was recommended by the WHO to utilize these three databases as they include $95 \%$ of the literature ${ }^{18}$. Key search terms were "Non-communicable diseases", "South Asia”, quality of care", accessibility, effectiveness, efficiency, acceptability, and "continuity of care".

\section{Screening}

Studies found from the primary search were exported to Endnote and two-step screening (title - abstract and full text) was done according to the inclusion and exclusion criteria. Citation tracking from the systematic review was done to include more studies in the review. A second reviewer checked and validated the screening process.

\section{Data extraction}

Data was extracted by using a detailed data extraction matrix where basic study information, intervention/approach taken, information on sample sizes, outcome variables, intervention details, relevant statistics, and information on outcomes, etc. were included. This matrix was piloted with two (10\%) studies to check the consistency.

\section{Bias assessment}

Cochrane risk of bias (ROB) tool was used for assessing Randomized Controlled Trials (RCTs) and Joanna Briggs Institute (JBI) Critical Appraisal tool was used to assess the quality of other studies. For overall grading of observational studies, 0-3 "yes" answers were marked as poor quality, 4-6 "yes" answers were considered as moderate quality, and more than six "yes" answers were ranked as a good quality study. Quality assessment was also validated by a second reviewer.

\section{Data synthesis}

A data-driven in-depth analysis was conducted through narrative synthesis, as there was considerable heterogeneity among the studies such as study design, methodology, intervention packages, outcome variables, results, and quality of the study ${ }^{18}$. A narrative synthesis was performed to arrange and compare relatively homogenous studies together according to their similarities and dissimilarities ${ }^{19,20}$. Meta-analysis was not conducted due to the absence of homogeneity among studies.

\section{Patient and public involvement}


This is a systematic review and no patients are directly involved in the process.

\section{Results:}

\section{Systematic review process}

Initially 829 studies were identified from three databases from which13 studies were considered for final analysis.

Figure: 1 describes how studies were found and included by applying the inclusion and exclusion criteria.

\section{Study setting and study characteristics}

Majority $(n=7)$ of included studies were from India followed by Pakistan $(n=2)$, and Bangladesh, Nepal, and Sri Lanka $(n=1)$. No studies were found from Afghanistan, Bhutan, or the Maldives. Most of the $(n=10)$ intervention took place at the hospital or clinic level ${ }^{21-30}$ whereas some $(n=3)$ were based on community setting ${ }^{31-33}$.

Majority of the included studies $(n=8)$ were cross-sectional $22,23,25,26,28,29,31,33$ followed by RCTs $(n=3){ }^{21,30,32}$, nonrandomized before-after intervention study $(n=1)(27)$ and qualitative study $(n=1)^{24}$ (Table 1). 
Table 1

Characteristics of included studies:

\begin{tabular}{|c|c|c|c|c|c|}
\hline Study identifier & Study Design & $\begin{array}{l}\text { Geographic } \\
\text { Location, } \\
\text { Population } \\
\text { age and } \\
\text { gender }\end{array}$ & NCD type & Intervention & Outcome/Result \\
\hline Ali et al., 2016 & $\begin{array}{l}\text { Parallel, open- } \\
\text { label, pragmatic } \\
\text { RCT }\end{array}$ & $\begin{array}{l}\text { - Diabetes } \\
\text { clinics in } \\
\text { India and } \\
\text { Pakistan } \\
\text { - } 35 \text { + years } \\
\text { - Male \& } \\
\text { Female }\end{array}$ & Diabetes & $\begin{array}{l}\text { Intervention: } \\
\text { Regular } \\
\text { physician's } \\
\text { care, diabetes } \\
\text { specific care } \\
\text { coordinator's } \\
\text { service through } \\
\text { monthly phone } \\
\text { call and follow- } \\
\text { up visit } \\
\text { once in three } \\
\text { months. } \\
\text { Control: Regular } \\
\text { physician's care } \\
\text { only. }\end{array}$ & 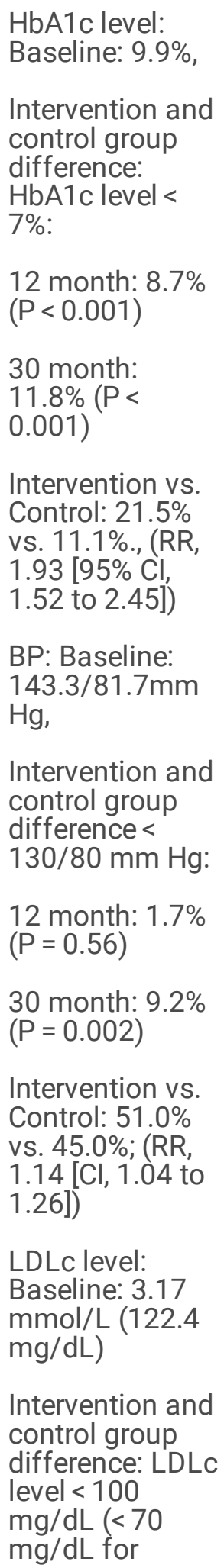 \\
\hline
\end{tabular}




\begin{tabular}{|c|c|c|c|c|c|}
\hline Study identifier & Study Design & $\begin{array}{l}\text { Geographic } \\
\text { Location, } \\
\text { Population } \\
\text { age and } \\
\text { gender }\end{array}$ & NCD type & Intervention & Outcome/Result \\
\hline & & & & & $\begin{array}{l}\text { people with } \\
\text { previous CVD): } \\
12 \text { month: } 9.5 \% \\
(P=0.001) \\
30 \text { month: } 5.3 \% \\
(P=0.071) \\
\text { Intervention vs. } \\
\text { Control: } 56.4 \% \\
\text { vs. } 47.1 \% \text {; (RR: } \\
1.23[\mathrm{Cl}, 1.13 \text { to } \\
1.34])\end{array}$ \\
\hline $\begin{array}{l}\text { Upadhyay et al., } \\
2015\end{array}$ & $\begin{array}{l}\text { Pre-post non- } \\
\text { clinical } \\
\text { randomised } \\
\text { controlled trial }\end{array}$ & $\begin{array}{l}\text { - Pokhara, } \\
\text { Nepal } \\
\text { - 16/+ years } \\
\text { - Male and } \\
\text { female both }\end{array}$ & $\begin{array}{l}\text { Diabetes } \\
\text { mellitus }\end{array}$ & $\begin{array}{l}\text { Test } 1 \text { group: } \\
\text { Educational } \\
\text { materials } \\
\text { (diabetes } \\
\text { information } \\
\text { booklet, } \\
\text { diabetes } \\
\text { complication } \\
\text { chart, diabetic } \\
\text { food chart, } \\
\text { exercise, using } \\
\text { insulin and } \\
\text { glucometer) for } \\
\text { increasing } \\
\text { patients' } \\
\text { diabetes } \\
\text { awareness and } \\
\text { management. } \\
\text { Test } 2 \text { group: } \\
\text { Educational } \\
\text { materials and } \\
\text { diabetic kit } \\
\text { (includes glass } \\
\text { tubing, chart of } \\
\text { human } \\
\text { anatomy with } \\
\text { circulatory } \\
\text { system, daily } \\
\text { medication } \\
\text { calendar and } \\
\text { calendar of } \\
\text { antidiabetic } \\
\text { medicines) } \\
\text { Control group: } \\
\text { Usual service } \\
\text { from nurse and } \\
\text { doctor }\end{array}$ & $\begin{array}{l}\text { Patients' } \\
\text { satisfaction } \\
\text { scores: } \\
\text { Control group: } \\
\text { Baseline: } 44 \\
\text { 3month: } 50 \\
12 \text { month: } 47.7 \\
\text { Test } 1 \text { group: } \\
\text { Baseline: } 45 \\
\text { 3month: } 66 \\
12 \text { month: } 68 \\
\text { Test } 2 \text { group: } \\
\text { Baseline: } 43 \\
\text { 3month: } 68 \\
12 \text { month: } 73\end{array}$ \\
\hline Lewis and Newell & Qualitative & - Dhaka & Type 2 & Discussed & - BIRDEM \\
\hline
\end{tabular}




\begin{tabular}{|c|c|c|c|c|c|}
\hline Study identifier & Study Design & $\begin{array}{l}\text { Geographic } \\
\text { Location, } \\
\text { Population } \\
\text { age and } \\
\text { gender }\end{array}$ & NCD type & Intervention & Outcome/Result \\
\hline et al, 2014 & study & $\begin{array}{l}\text { metropolitan } \\
\text { City \& Sylhet } \\
\text { division, } \\
\text { Bangladesh } \\
\text { - Age group } \\
\text { not } \\
\text { mentioned } \\
\text { - Male and } \\
\text { female }\end{array}$ & Diabetes & $\begin{array}{l}\text { about available } \\
\text { diabetes care in } \\
\text { different setting } \\
\text { in Bangladesh. } \\
\text { Control group: } \\
\text { N/A }\end{array}$ & $\begin{array}{l}\text { provides } \\
\text { comprehensive } \\
\text { education } \\
\text { program (verbal } \\
\text { and written) on } \\
\text { diabetes care } \\
\text { and patients } \\
\text { have good } \\
\text { awareness. } \\
\text { - Only the } \\
\text { BIRDEM clinic } \\
\text { situated in } \\
\text { capital offered } \\
\text { regular } \\
\text { comprehensive } \\
\text { check-ups (full } \\
\text { cardiovascular, } \\
\text { renal and } \\
\text { eyesight } \\
\text { examinations). } \\
\text { - Limited } \\
\text { knowledge } \\
\text { among patients } \\
\text { who take } \\
\text { service from } \\
\text { specialist } \\
\text { centres due to } \\
\text { lack of getting } \\
\text { proper diabetes } \\
\text { guideline. } \\
\text { - Rural Upazilla- } \\
\text { level clinics } \\
\text { contains limited } \\
\text { resources to } \\
\text { manage } \\
\text { diabetes. } \\
\text {-High service } \\
\text { cost, resource } \\
\text { limitation, long } \\
\text { waiting line } \\
\text { creates } \\
\text { limitation to } \\
\text { provide } \\
\text { comprehensive } \\
\text { treatment for } \\
\text { service } \\
\text { providers. } \\
\text { - Poor diabetes } \\
\text { management in } \\
\text { rural and peri- } \\
\text { urban area due } \\
\text { to high service } \\
\text { cost. }\end{array}$ \\
\hline
\end{tabular}




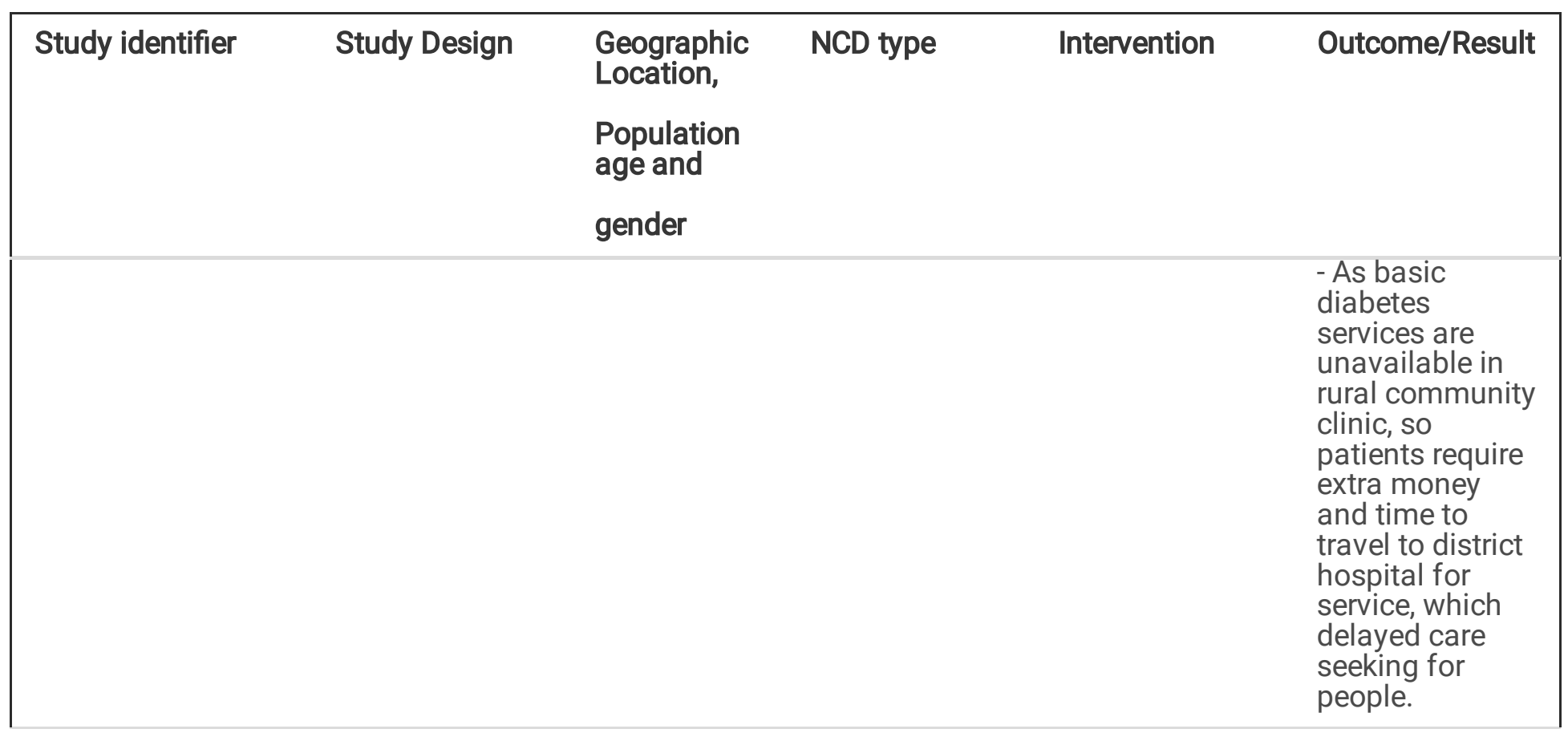




\begin{tabular}{|c|c|c|c|c|c|}
\hline Study identifier & Study Design & $\begin{array}{l}\text { Geographic } \\
\text { Location, } \\
\text { Population } \\
\text { age and } \\
\text { gender }\end{array}$ & NCD type & Intervention & Outcome/Result \\
\hline Sing et al. 2014 & $\begin{array}{l}\text { Cross sectional } \\
\text { study }\end{array}$ & $\begin{array}{l}\text { - } \\
\text { India } \\
\text { - Mean age } \\
31.49 \\
\text { - Gender not } \\
\text { mentioned }\end{array}$ & $\begin{array}{l}\text { Cardiovascular } \\
\text { disease }\end{array}$ & $\begin{array}{l}\text { Intervening } \\
\text { mobile phone } \\
\text { and Bluetooth } \\
\text { operated } \\
\text { handheld tele- } \\
\text { ECG machine in } \\
\text { community } \\
\text { level. } \\
\text { Control group: } \\
\text { N/A }\end{array}$ & $\begin{array}{l}\text { - 100\% } \\
\text { accuracy of } \\
\text { transmission } \\
\text { rate of tele-ECG } \\
\text { from handheld } \\
\text { machine to } \\
\text { mobile phone. } \\
\text { - Tele-ECG result } \\
\text { was transmitted } \\
\text { to expert } \\
\text { physicians } \\
\text { based in } \\
\text { Postgraduate } \\
\text { Institute of } \\
\text { Medical } \\
\text { Education and } \\
\text { Research } \\
\text { (PGIMER) from } \\
\text { remote area } \\
\text { - Patients with } \\
\text { acute } \\
\text { myocardial } \\
\text { infraction were } \\
\text { screened } \\
\text { through the tele- } \\
\text { ECG machine } \\
\text { and got } \\
\text { immediate } \\
\text { service from the } \\
\text { specialized } \\
\text { doctor. } \\
\text { - Patients } \\
\text { reported } ~ 95 \% \\
\text { satisfaction } \\
\text { about new tele- } \\
\text { ECG machine as } \\
\text { it ensured the } \\
\text { availability of } \\
\text { health care for } \\
\text { people who } \\
\text { lives in remote } \\
\text { area. }\end{array}$ \\
\hline
\end{tabular}




\begin{tabular}{|c|c|c|c|c|c|}
\hline Study identifier & Study Design & $\begin{array}{l}\text { Geographic } \\
\text { Location, } \\
\text { Population } \\
\text { age and } \\
\text { gender }\end{array}$ & NCD type & Intervention & Outcome/Result \\
\hline Basu et al., 2006 & $\begin{array}{l}\text { Cross sectional } \\
\text { study }\end{array}$ & $\begin{array}{l}\text { - Rural } \\
\text { district of } \\
\text { Bengal, a } \\
\text { state in } \\
\text { eastern } \\
\text { India. } \\
-30-65 \\
\text { years } \\
\text { - Female }\end{array}$ & Cervical cancer & $\begin{array}{l}\text { Community } \\
\text { based cervical } \\
\text { cancer } \\
\text { screening test/ } \\
\text { via-test for } \\
\text { women aged } \\
30-65 \text { years. } \\
\text { Control group: } \\
\text { N/A }\end{array}$ & $\begin{array}{l}\text { - Immediate } \\
\text { colposcopy for } \\
\text { women with } \\
\text { positive via } \\
\text { screening: } 100 \% \\
\text { compliance } \\
\text { - Cervical punch } \\
\text { biopsies for } \\
\text { women with } \\
\text { abnormal } \\
\text { colposcopy: } \\
95.6 \% \\
\text { compliance, } \\
\text { Biopsy was } \\
\text { refused by } 7 \\
\text { women. } \\
\text { - Satisfied and } \\
\text { very satisfied } \\
\text { with the service: } \\
64.7 \% \text { \& } 5.6 \% \\
\text { accordingly } \\
\text { - Accessibility } \\
\text { and } \\
\text { affordability } \\
\text { mentioned by } \\
\text { service recipient } \\
\text { as it was } \\
\text { community } \\
\text { based and free } \\
\text { screening. }\end{array}$ \\
\hline $\begin{array}{l}\text { Sankaranarayanan } \\
\text { et al., } 2012\end{array}$ & $\begin{array}{l}\text { Cluster } \\
\text { randomized } \\
\text { controlled trial }\end{array}$ & $\begin{array}{l}\text { - Trivandrum } \\
\text { district, } \\
\text { Kerala, } \\
\text { India. } \\
\text { - 35/+ years } \\
\text { - Male \& } \\
\text { Female }\end{array}$ & Oral Cancer & $\begin{array}{l}\text { Intervention: } \\
\text { Health worker } \\
\text { provided } \\
\text { screening } \\
\text { facilities for } \\
\text { oral cancer and } \\
\text { health } \\
\text { education to } \\
\text { quit harmful } \\
\text { practices + } \\
\text { further direction } \\
\text { on treatment } \\
\text { from specialist } \\
\text { for those who } \\
\text { are screened } \\
\text { positive. } \\
\text { Control: Routine } \\
\text { health care } \\
\text { without } \\
\text { screening } \\
\text { facilities until } \\
2006\end{array}$ & $\begin{array}{l}\text { - Cumulative } \\
\text { advanced oral } \\
\text { cancer mortality } \\
\text { rate: RR 0.88, } \\
95 \% \mathrm{Cl}(0.69- \\
1.12) \\
\\
\text { - Incidence of } \\
\text { advanced oral } \\
\text { cancers among } \\
\text { tobacco/alcohol } \\
\text { user or both: RR } \\
0.79,95 \% \mathrm{Cl} \\
(0.65-0.95) \\
\text { - Advanced oral } \\
\text { cancer mortality } \\
\text { among } \\
\text { tobacco/alcohol } \\
\text { user or both: RR } \\
0.76,95 \% \mathrm{Cl} \\
\text { (0.60-0.97) }\end{array}$ \\
\hline
\end{tabular}




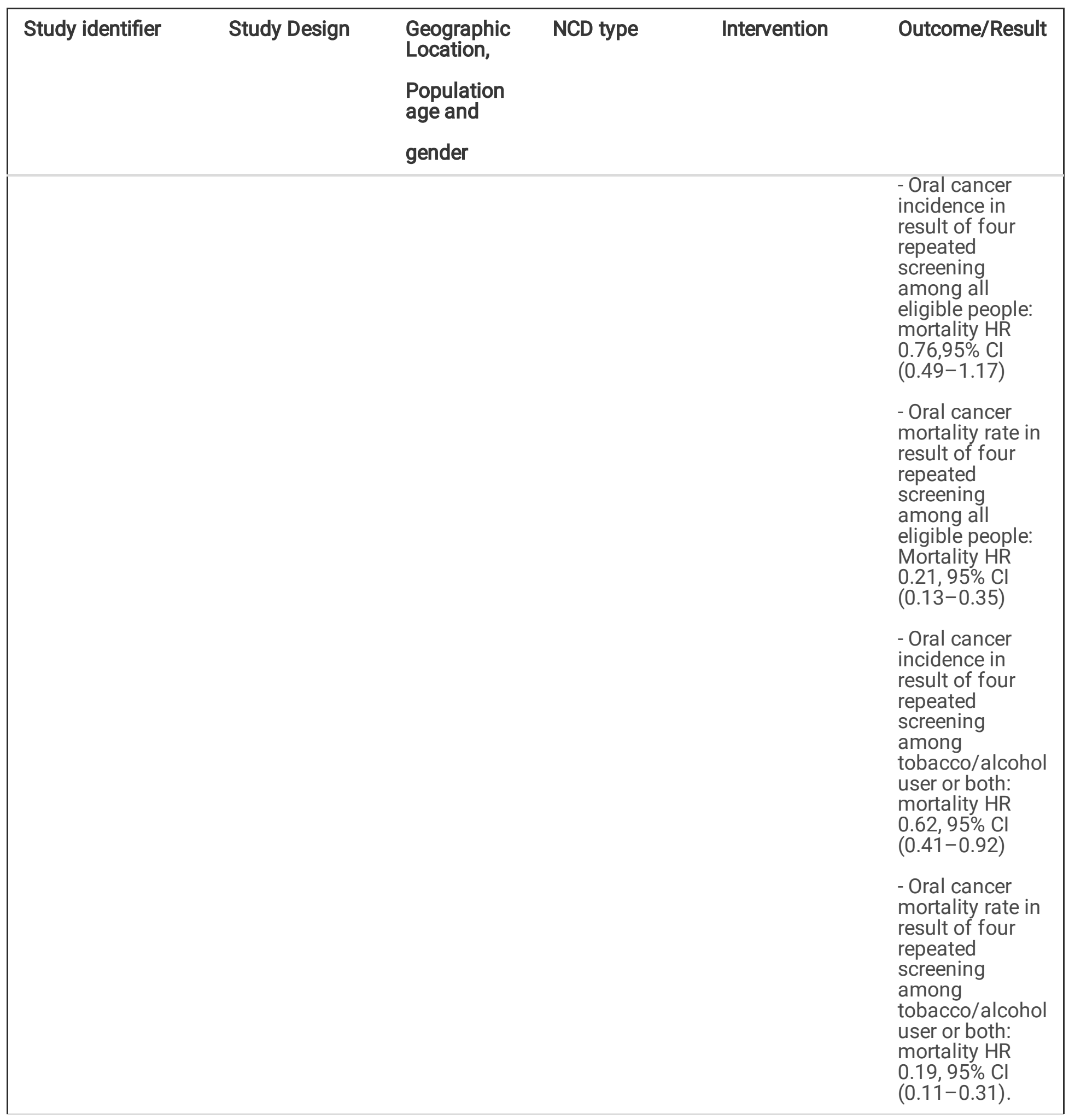




\begin{tabular}{|c|c|c|c|c|c|}
\hline Study identifier & Study Design & $\begin{array}{l}\text { Geographic } \\
\text { Location, } \\
\text { Population } \\
\text { age and } \\
\text { gender }\end{array}$ & NCD type & Intervention & Outcome/Result \\
\hline $\begin{array}{l}\text { Mahapatra et al., } \\
2016\end{array}$ & $\begin{array}{l}\text { Crosssectional } \\
\text { study }\end{array}$ & $\begin{array}{l}\text { - Odisha, } \\
\text { India } \\
-21-40 \\
\text { years } \\
\text { - Male and } \\
\text { female }\end{array}$ & Cancer & $\begin{array}{l}\text { Oncology } \\
\text { services } \\
\text { provision in } \\
\text { specialty } \\
\text { hospitals in } \\
\text { Odisha, India. } \\
\text { Control group: } \\
\text { N/A }\end{array}$ & $\begin{array}{l}\text { - } 13 \text { out of } 22 \\
\text { patients } \\
\text { reported about } \\
\text { good } \\
\text { interpersonal } \\
\text { behaviour of } \\
\text { doctors. } \\
\text { However, } \\
\text { negative } \\
\text { behaviour from } \\
\text { supporting staff } \\
\text { was reported. } \\
\text { - Patient } \\
\text { satisfaction on } \\
\text { interpersonal } \\
\text { manner } 63 \% \\
\text { (3.2 } \pm 0.5 \text { ). } \\
\text { - Patient } \\
\text { satisfaction in } \\
\text { overall } \\
\text { communication } \\
\text { 70\% (3.3 } 0.5 \text { ). } \\
\text { - Few problems } \\
\text { such as long } \\
\text { waiting hours, } \\
\text { shortage of bed } \\
\text { for admission, } \\
\text { long distance of } \\
\text { specialized } \\
\text { hospital etc. } \\
\text { were reported. }\end{array}$ \\
\hline $\begin{array}{l}\text { Chiranthika et al., } \\
2013\end{array}$ & $\begin{array}{l}\text { Cross-sectional } \\
\text { study }\end{array}$ & $\begin{array}{l}\text { - Gampaha, } \\
\text { Western } \\
\text { province of } \\
\text { Sri Lanka } \\
\text { - 35-39 } \\
\text { years } \\
\text { - Female }\end{array}$ & Breast cancer & $\begin{array}{l}\text { Clinic based } \\
\text { early detection } \\
\text { service for } \\
\text { breast cancer } \\
\text { were provided. } \\
\text { Then, } \\
\text { assessment } \\
\text { was done on } \\
\text { coverage, } \\
\text { quality and } \\
\text { client } \\
\text { satisfaction. } \\
\text { Control group: } \\
\text { N/A }\end{array}$ & $\begin{array}{l}\text { Coverage: } \\
\text { - Clinical Breast } \\
\text { Examination } \\
\text { coverage } \\
\text { increased from } \\
1.1 \%-2.2 \% \\
\text { between } 2003- \\
2007 . \\
\text { - Proportion of } \\
\text { breast } \\
\text { abnormalities } \\
\text { detected on } \\
2007: 1.8 \% \text {. } \\
\\
\text { - Proportion } \\
\text { referred for } \\
\text { further care } \\
\text { detected with } \\
\text { breast } \\
\text { abnormalities: } \\
86.8 \% \text {. }\end{array}$ \\
\hline
\end{tabular}




\begin{tabular}{|c|c|c|c|c|c|}
\hline Study identifier & Study Design & $\begin{array}{l}\text { Geographic } \\
\text { Location, } \\
\text { Population } \\
\text { age and } \\
\text { gender }\end{array}$ & NCD type & Intervention & $\begin{array}{l}\text { Ouality: } \\
\text { - Clients } \\
\text { satisfaction } \\
\text { with the } \\
\text { infrastructure: } \\
\text { - Space in the }\end{array}$ \\
\hline & & & & & $\begin{array}{l}\text { clinic building: } \\
83 \% \text {, } \\
\text { - Overall } \\
\text { cleanliness of } \\
\text { clinic: } 82.5 \% \text {, } \\
\text { - Cleanliness of } \\
\text { the } \\
\text { toilets:58.5\%, } \\
\text { - Availability of } \\
\text { sitting facilities } \\
\text { in the waiting } \\
\text { area: } 85.5 \% \text {, } \\
\text { - Comfort in the } \\
\text { waiting area: } \\
84.5 \% \\
\text { Satisfaction on } \\
\text { service } \\
\text { provision: } \\
\text { - Politeness } \\
\text { displayed by the } \\
\text { health care } \\
\text { workers: } 98 \% \text {, } \\
\text { - Privacy while } \\
\text { conducting CBE: } \\
86 \% \text {, } \\
\text { - Time spent on } \\
\text { CBE: } 97 \% \text {, } \\
\text { - Health } \\
\text { education on } \\
\text { BSE: } 98 \%\end{array}$ \\
\hline $\begin{array}{l}\text { Mathew et al., } \\
2017\end{array}$ & $\begin{array}{l}\text { Cross sectional } \\
\text { study }\end{array}$ & $\begin{array}{l}\text { - Mumbai, } \\
\text { India } \\
-30-79 \\
\text { years } \\
\text { - Male and } \\
\text { female }\end{array}$ & 更 & $\begin{array}{l}\text { Telephonic } \\
\text { follow-up for } \\
\text { cancer patients } \\
\text { with planned } \\
\text { treatment was } \\
\text { introduced. } \\
\text { Control group: } \\
\text { N/A }\end{array}$ & $\begin{array}{l}\text { - Agreement } \\
\text { between the } \\
\text { telephonic and } \\
\text { physical } \\
\text { impression of } \\
\text { disease: } \\
\text { Substantial } \\
\text { strength } \\
\text { - Accuracy of } \\
\text { telephonic } \\
\text { versus physical } \\
\text { follow-up: } \\
\text { Among seven } \\
\text { follow-up, five } \\
\text { showed } \\
\text { substantial } \\
\text { strength (PABAK }\end{array}$ \\
\hline
\end{tabular}




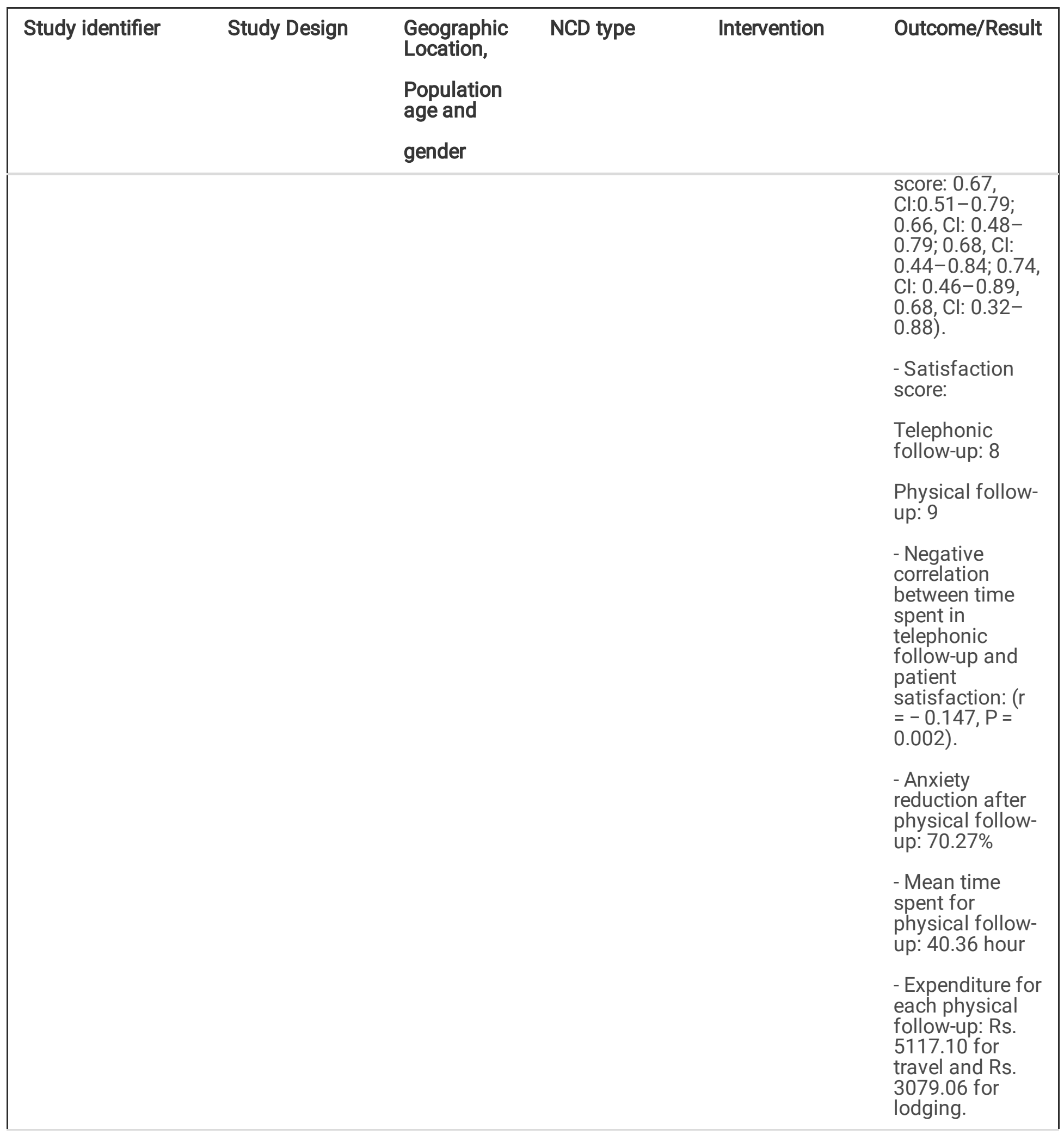




\begin{tabular}{|c|c|c|c|c|c|}
\hline Study identifier & Study Design & $\begin{array}{l}\text { Geographic } \\
\text { Location, } \\
\text { Population } \\
\text { age and } \\
\text { gender }\end{array}$ & NCD type & Intervention & Outcome/Result \\
\hline $\begin{array}{l}\text { Ghoshal et al. } \\
2019\end{array}$ & $\begin{array}{l}\text { Cross-sectional } \\
\text { study }\end{array}$ & $\begin{array}{l}\text { - India } \\
\text { - >= } 18 \text { years } \\
\text { - Male and } \\
\text { female }\end{array}$ & Cancer & $\begin{array}{l}\text { Advanced } \\
\text { cancer patients' } \\
\text { decision } \\
\text { making about } \\
\text { treatment were } \\
\text { measured in a } \\
\text { palliative care } \\
\text { unit. } \\
\text { Control group: } \\
\text { N/A }\end{array}$ & $\begin{array}{l}\text { - Shared, active, } \\
\text { and passive } \\
\text { Decisional } \\
\text { Control } \\
\text { Preferences } \\
\text { (DCP) was } \\
20.7 \%, 26.7 \% \text {, } \\
\text { and } 52.7 \% \text {, } \\
\text { respectively. } \\
\text { - } 27.3 \% \text { felt that } \\
\text { the doctor } \\
\text { should make a } \\
\text { shared decision } \\
\text { with the patient, } \\
\text { - } 34 \% \text { patients } \\
\text { felt that the } \\
\text { family should } \\
\text { be involved in } \\
\text { decision } \\
\text { making. } \\
\text { - } 32.7 \% \text { make } \\
\text { the decisions } \\
\text { with the family } \\
\text { after consulting } \\
\text { with the doctor. } \\
\text { - } 59.3 \% \text { actual } \\
\text { treatment } \\
\text { decisions were } \\
\text { passive, } \\
\text { whereas } 21.3 \% \\
\text { were actively } \\
\text { taken by the } \\
\text { patient. }\end{array}$ \\
\hline Shams et al., 2018 & $\begin{array}{l}\text { Cross sectional } \\
\text { study }\end{array}$ & $\begin{array}{l}\text { - Karachi, } \\
\text { Pakistan. } \\
-20-60 \text { + } \\
\text { years } \\
\text { - Female }\end{array}$ & $\begin{array}{l}\text { Breast and } \\
\text { gynaecological } \\
\text { cancer }\end{array}$ & $\begin{array}{l}\text { Intervention } \\
\text { group: } \\
\text { Structured } \\
\text { supportive care } \\
\text { (physical and } \\
\text { psychosocial } \\
\text { counselling, } \\
\text { mind diversion } \\
\text { activities) for } \\
\text { patients taking } \\
\text { chemotherapy } \\
\text { for } 6 \text { weeks. } \\
\text { Control group: } \\
\text { N/A }\end{array}$ & $\begin{array}{l}\text { - Improved } \\
\text { selfcare } \\
\text { behaviour, } \\
\text { physical and } \\
\text { psychological } \\
\text { health and } \\
\text { satisfaction } \\
\text { among the } \\
\text { intervention } \\
\text { participants. } \\
\text { - Almost all } \\
\text { participants } \\
\text { were satisfied } \\
\text { with the } \\
\text { program. } \\
\text { - Intervention } \\
\text { gave emotional } \\
\text { support and }\end{array}$ \\
\hline
\end{tabular}




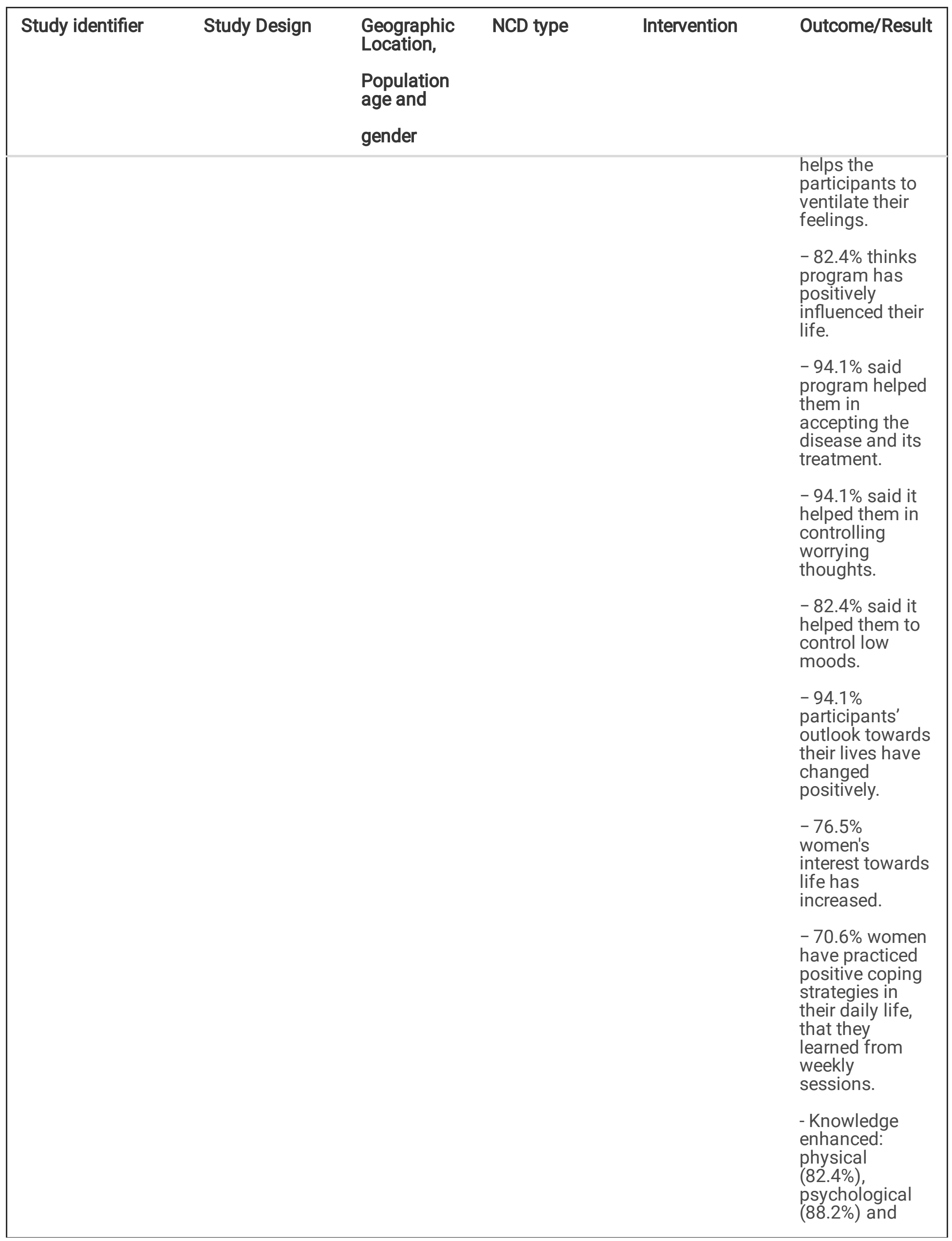




\begin{tabular}{|c|c|c|c|c|c|}
\hline Study identifier & Study Design & $\begin{array}{l}\text { Geographic } \\
\text { Location, } \\
\text { Population } \\
\text { age and } \\
\text { gender }\end{array}$ & NCD type & Intervention & Outcome/Result \\
\hline & & & & & $\begin{array}{l}\text { sexual health } \\
(76.5 \%)\end{array}$ \\
\hline Nayak et al., 2005 & $\begin{array}{l}\text { Nonrandomized } \\
\text { Before-after } \\
\text { intervention } \\
\text { study }\end{array}$ & $\begin{array}{l}\text { - Cuttack, } \\
\text { India } \\
\text { - Pre: } 28-79 \\
\text { years } \\
\text { - Post: } 23- \\
81 \text { years } \\
\text { - Male and } \\
\text { female }\end{array}$ & 更 & $\begin{array}{l}\text { Communication } \\
\text { strategy for } \\
\text { service } \\
\text { providers } \\
\text { developed and } \\
\text { implemented } \\
\text { Control group: } \\
\text { N/A }\end{array}$ & $\begin{array}{l}\text { - Allowing } \\
\text { enough time for } \\
\text { the patient and } \\
\text { families } \\
\text { 1st step: } 22 \% \text {, } \\
\text { 3rd step: } 42 \% \text { (p } \\
\text { <.001) } \\
\text { - Doctor's } \\
\text { attitude towards } \\
\text { clarification of } \\
\text { issues } \\
\text { 1st step: } 26 \% \text {, } \\
\text { 3rd step: } 56 \% \text { (p } \\
\text { <.001) } \\
\text { - Use of clear } \\
\text { language } \\
\text { 1st step: 14\%, } \\
\text { 3rd step: } 57 \% \text { ( } \\
\text { p<.001) } \\
\text {-Privacy during } \\
\text { consultation } \\
\text { 1st step: } 5 \% \text {, 3rd } \\
\text { step: } 70 \% \text { ( } p \\
<.001 \text { ) } \\
\text {-No interruption } \\
\text { during } \\
\text { consultation } \\
\text { 1st step: } 42 \% \text {, } \\
\text { 3rd step: } 82 \% \text { ( } \\
\text { p<.001) } \\
\text { - Overall } \\
\text { satisfaction } \\
\text { with } \\
\text { communication } \\
\text { 1st step: 13\%, } \\
\text { 3rd step: } 33 \% \text { ( } \\
\text { p<.001). }\end{array}$ \\
\hline
\end{tabular}




\begin{tabular}{|c|c|c|c|c|c|}
\hline Study identifier & Study Design & $\begin{array}{l}\text { Geographic } \\
\text { Location, } \\
\text { Population } \\
\text { age and } \\
\text { gender }\end{array}$ & NCD type & Intervention & Outcome/Result \\
\hline Tovey et al., 2005 & $\begin{array}{l}\text { Cross-sectional } \\
\text { study }\end{array}$ & $\begin{array}{l}\text { - Lahore, } \\
\text { Pakistan } \\
\text { - Age group } \\
\text { not } \\
\text { mentioned } \\
\text { - Male and } \\
\text { female }\end{array}$ & Cancer & $\begin{array}{l}\text { Cancer patients } \\
\text { were asked in } \\
\text { four different } \\
\text { hospitals about } \\
\text { their } \\
\text { satisfaction } \\
\text { towards using } \\
\text { traditional } \\
\text { medicine (TM) } \\
\text { and } \\
\text { Complimentary } \\
\text { Alternative } \\
\text { Medicine (CAM) } \\
\text { beside } \\
\text { allopathic } \\
\text { medicine. } \\
\text { Control group: } \\
\text { N/A }\end{array}$ & $\begin{array}{l}\text { - Most used } \\
\text { CAM/TMs by } \\
\text { cancer patients' } \\
\text { in Pakistan are } \\
\text { Dam Darood } \\
\text { (70.4\%), and } \\
\text { spiritual healing } \\
\text { (47.2\%) and } \\
\text { Hakeem (35\%). } \\
\text { - } 84 \% \text { of the } \\
\text { cancer patients } \\
\text { had used } 1 \text { or } \\
\text { more forms of } \\
\text { TM in } \\
\text { combination } \\
\text { with } \\
\text { conventional } \\
\text { treatments. } \\
\text { - To the patients, } \\
\text { CAM/TMs are } \\
\text { also thought to } \\
\text { be effective and } \\
\text { very effective } \\
\text { (Dam Darood } \\
57 \% \text {, spiritual } \\
\text { healing } 26 \% \\
\text { and Hakeem } \\
22 \%) \text { beside } \\
\text { medical } \\
\text { specialists } \\
\text { (94\%) and } \\
\text { general } \\
\text { practitioners } \\
\text { (78\%). }\end{array}$ \\
\hline & & & & & $\begin{array}{l}\text { - } 58 \% \text { patients } \\
\text { were satisfied } \\
\text { with the cancer } \\
\text { treatment of } \\
\text { homeopathy. }\end{array}$ \\
\hline
\end{tabular}

\section{Quality assessment}

Among the RCTs, allocation concealment was found "unclear" for two studies ${ }^{30,32}$ random sequence generation, performance bias, detection bias, and attrition bias was found as low risk of bias for two studies. All studies reported a low risk of reporting bias $21,30,32$. The absence of allocation concealment and attrition rate resulted in the unclear risk of bias for one study ${ }^{32}$. The remaining RCT did not mention any of the bias except the primary outcome and attrition rate ${ }^{30}$. The risk of bias of RCTs has been demonstrated in Fig. 2. 
In overall grading, among eight cross-sectional studies, four studies $22,23,26,31$ were of good quality, three ${ }^{25,28,33}$ were of moderate quality and one study ${ }^{29}$ was found to have poor quality. As per the adapted grading system, the qualitative study ${ }^{24}$ and non-randomized before-after intervention study ${ }^{27}$ were graded as the good quality study as well. Studies that were assessed as a good quality study had an adequate answer for study setting, sample size and sampling method, valid and reliable statistical analysis, and reporting system, ethical consideration and managing confounding.

\section{Intervention and outcome:}

\section{Intervention related to cancer and its outcome}

9 out of 13 studies that were included in this review focused on interventions to improve QoC in cancer services. Here, two studies discussed community-based ${ }^{31,32}$, and one study discussed clinic-based screening services ${ }^{22}$ to improve QoC for cancer patients.

A community-based cervical cancer screening program by trained health workers (HW) was discussed in one study where Visual Inspection with Acetic Acid (VIA) test, colposcopy, punch biopsy, treatment, and advice were provided consecutively which increases the availability of service provision to village women and treatment compliance and reduce the incidence and severity of advanced cervical cancer. $64.7 \%$ of women were satisfied while $5.6 \%$ were very satisfied with the services. Women with positive results on the VIA test were immediately scheduled for colposcopy and women with abnormal colposcopy were referred for cervical punch biopsies which result in 100\% and $95.6 \%$ compliance to treatment in both cases ${ }^{31}$.

15 years long RCT on community-based oral cancer screening program was run by trained HW where awareness rising through home visits, screening services, further treatment for positively screened patients, referrals, etc. were provided to the community people to increase treatment facilities and awareness ${ }^{32}$. This intervention significantly reduced the mortality rate of oral cancer among tobacco/ alcohol users and non-users. Through community-based care and health education, the incidence of advanced oral cancer among tobacco/alcohol users reduced significantly (RR $0.79,95 \% \mathrm{Cl}: 0.65-0.95)^{32}$.

A clinic-based screening and early detection program was conducted in Gampaha district, Sri Lanka, where clinical breast examination (CBE) and breast self-examination (BSE) was done by public health midwives/HW for early detection of breast cancer. Women were found satisfied with clinic space (83\%), privacy (86\%) and time spent (97\%) during CBE, toilet cleanliness (58.5\%), availability and comfort of sitting facilities in the waiting area (85.5\% and $84.5 \%)$. Clinic-based breast cancer detection program has helped to increase breast cancer detection and service coverage from $1.1 \%-2.2 \%$ between $2003-2007$. Referral service increase and $86.8 \%$ of women who were detected with breast abnormalities were referred for further care ${ }^{22}$.

In one study, telephonic follow-up beside physical follow-up was arranged for the patients with cancer who were already under planned treatment. An unchanged questionnaire was used to compare the patient's physical condition, satisfaction, cost, and time associated with both types of follow-up. A negative correlation was found between time spent in telephonic follow-up and patient satisfaction $(r=-0.147, P=0.002)$. Patients preferred a physical follow-up even though it required a higher financial investment. $70.27 \%$ of study participants acknowledged the reduction in anxiety when they attended a physical follow-up ${ }^{26}$. 
Due to a structured five weeklong hospital-based physical and psychological supportive care for women undergoing chemotherapy for six weeks, patient's ability to control their mood (82.4\%), acceptance of the disease and its treatment $(94.1 \%)$, positive outlook toward life (76.5\% women's interest toward life grows and $70.6 \%$ of women to practice positive coping strategies in their daily life), etc. increases. This intervention was packed with group therapy where physical and psychosocial counselling and mind diversion activities for improving psychological and emotional wellbeing were provided ${ }^{28}$.

It was explored through one study that improved communication skills of service providers can increase patient's satisfaction around their behaviour. Three staged-intervention of this study included investigation of the patient's perception of doctor-patient communication, dissemination of results with service providers, and development of a communication strategy for service providers and provision of training for them 27 .

Two studies discussed how a specialized hospital and palliative care centre for cancer could serve patients in a more engaging way ${ }^{25}$ and how the decision-making process around treatment could be influenced ${ }^{34}$. Services from a specialized cancer hospital had increased patient satisfaction (63\%) related to the interpersonal relationship with their service provider $(3.2 \pm 0.5)$. $70 \%$ of patients were also satisfied with the overall communication $(3.3 \pm 0.5)$ even though issues such as long waiting times, shortage of beds and, long-distance to a specialized hospital are present 25 . It was identified that a specialized palliative care unit could help patients with making decisions related to treatment by consulting with the specialized doctor ${ }^{34}$.

\section{- Intervention related to diabetes and its outcome:}

Three among thirteen studies discussed interventions that ensure QoC around diabetic care. For maintaining QoC in service provision the included studies discussed different approaches such as employing diabetic care specific HW, providing diabetic education and care, and initiating specialized service for diabetes $21,24,30$.

It was found through a 2.5-year-long RCT ${ }^{21}$, that HWs specialized in diabetic care resulted in improved and continuous care. This RCT employed diabetes care specific HW who communicated with a diabetic patient from the intervention group every month over the phone and organized a follow-up visit once every three months, in addition to regular visits to the physician. HWs also developed a management plan for patients by looking at patients' laboratory tests, treatment plans, and discuss them with the doctors. Because of diabetes care specific HW's patient follow-up and care, patients of the intervention group achieved the study objective ${ }^{21}$.

Another RCT conducted in Nepal had three arms where two were intervention arms (named as test 1 and test 2 group) and one was a control arm ${ }^{30}$. Both intervention groups received diabetes-related education materials, whereas participants in test 2 received diabetic kits along with educational materials. Due to this intervention, the satisfaction score among the intervention group has increased after 12 months when compared to the control group. The second intervention group received an extra diabetic kit along with education materials, which increased their satisfaction scores even more ${ }^{30}$.

Through a qualitative study conducted by Lewis and Newell discussed the available diabetic care from BIRDEM, BADAS, district health complexes, community clinics in the rural area, and the slums of Bangladesh ${ }^{24}$. Unlike BADAS, BIRDEM provides a comprehensive awareness program, written and verbal information. It also discussed the limitations of a specialized center for diabetes care in Bangladesh and the high cost of diabetes care outside of BIRDEM. They emphasized the lack of access to clinics with appropriate facilities for diabetic care for people living 
in rural areas and subdistrict level. Specialized diabetes center could raise awareness on diabetes management and the availability of comprehensive check-ups (full cardiovascular, renal, and eyesight examinations) and proper health education, could minimize the risk for future complications in diabetic patients ${ }^{24}$.

\section{- Intervention related to CVD and its outcome:}

To maintain QoC around CVD a study included in this review discussed a community-based screening service where a unique handheld tele-ECG machine was used. As early diagnosis and screening of CVD are difficult for people living in rural areas, community-based screening services with tele-ECG machines screened patients with acute myocardial infarction and they got immediate service from a specialized doctor. It achieved a $95 \%$ satisfaction rate for people living in remote areas as well ${ }^{33}$.

\section{Discussions:}

Among four major NCDs, approaches around three NCDs (cancer, diabetes, CVD) were derived from the search, and studies related to Chronic respiratory disease was not retrieved in this review. The most important approach found from included studies of this review were community-based screening and early detection, community-based mHealth, patient follow-up, and health education through HW, the establishment of specialized hospital or corner for NCDs, communication training for service providers, and services that provide extra support for patients.

This review found that availability of services (screening, early detection, and, regular check-up) near to the community increase the accessibility, affordability and patient satisfaction ${ }^{31-33}$ which is similar to a study conducted in Malawi, that showed the lack of accessibility, affordability of transportation, service fee, and, poverty hinder the uptake of NCD services. Therefore, to ensure QoC, NCD services need to be close to the community so that people can have improved access to these services and allow it to be more equitable for women and the poor ${ }^{35}$.

It was also found through this review that community-based screening services allow patients to have the opportunity to detect existing diseases and seek next level treatment if needed ${ }^{31}$. These findings are also supported by a review conducted by Kösters and Gøtzsche, that breast cancer screening was associated with an estimated $88 \%$ increase in seeking a biopsy compared to the control group (RR $1.88,95 \% \mathrm{Cl} 1.77-1.99){ }^{36}$.

This review suggested a strong approach to ensure QoC around diabetic care which is HW associated with patient follow-up and health education provision 21,30 . A systematic review done in sub-Saharan Africa found similar findings, where counselling and health education on diet, exercise, and smoking cessation significantly decreased HbA1c levels from $10.6+/-4.2 \%$ to $7.6+/-2.3 \%(p<0.001){ }^{37}$.

Comprehensive education programs and booklet distribution (verbal and written) on NCDs were also found as strong instruments to maintain QoC in this review ${ }^{24}$ which is aligned with a patient-centered education intervention through booklet distribution among heart failure patients in Mulago Hospital, Kampala, Uganda. It showed positive improvements in patient satisfaction ( $16 \%$ to $79 \%, p<0 \cdot 001)$, awareness of their present health condition $(80 \%$, $p<0.0001)$, self-care management, and disease prevention $(83 \%, p<0.0001)$ came due to the intervention ${ }^{38}$.

The current review revealed that maintaining patient privacy, confidentiality, and adequate time with the patient during the consultation is crucial for ensuring patient satisfaction ${ }^{27}$ which are also comparable with a systematic review on family planning conducted in the African context ${ }^{39}$. This review suggested provision of communication 
training for the service provider can increase client satisfaction which is important to maintain QoC in service provision ${ }^{27}$. A study that took place in Kazakhstan also recommended the same for improving doctor-patient relationships for better adherence to treatment ${ }^{40}$.

Despite having limited evidence in QoC around NCD services in South Asia, this review provides few practical research implications. Community and clinic-based services ornate with the referral, regular follow-up might ensure QoC. In addition to this, NCD related health education, setting up specialized NCD services could be other options. Besides, more research on this field is required as the limited number of experimental studies has been derived from this review.

South Asian countries have a high NCD burden yet QoC around NCD services have been neglected. The interventions for improving QoC around NCD services described in this review could be scaled up in different settings in South Asia. Multiple stakeholders across different sectors could focus on this review to further implement health programs and alleviate the burden of NCDs in South Asia.

\section{List Of Abbreviations}

COPD Chronic obstructive pulmonary disease

JBI Joanna Briggs Institute

LMICs Low and middle-income countries

NCDs Non-communicable diseases

PRISMA Preferred reporting items for systematic review and meta-analysis

PROSPERO International prospective register of systematic reviews

PHC Primary health care

QoC Quality of care

RCTs Randomized controlled trials

SDG Sustainable development goal

UHC Universal health coverage

WHO World Health Organization

\section{Declarations}

\section{Ethics approval and consent to participate:}

Not applicable.

\section{Consent for publication:}




\section{Availability of data and materials:}

All data generated or analysed during this study are included in this published article (and its supplementary information files).

\section{Competing interests:}

None declared.

\section{Funding:}

There was no funding for this research.

\section{Authors' contributions:}

SE and KMSUR conceptualized the systematic review. SE drafted the manuscript with potential inputs from KMSUR. KMSUR critically reviewed the manuscript. All authors revised and approved the final manuscript.

\section{Acknowledgements:}

The authors would like to acknowledge the contribution of the current donors providing unrestricted support to icddr,b that include: the Governments of Bangladesh, Canada, Sweden and the UK. We gratefully acknowledge these donors for their support and commitment to icddr,b's research efforts.

\section{References}

1. WHO. Global Health Observatory (GHO) data: NCD mortality and morbidity 2019. Available from: https://www.who.int/gho/ncd/mortality_morbidity/en/.

2. WHO. Noncommunicable diseases country profiles 2018. Geneva: World Health Organization; 2018.

3. Mallath MK, Taylor DG, Badwe RA, Rath GK, Shanta V, Pramesh C, et al. The growing burden of cancer in India: epidemiology and social context. The Lancet Oncology. 2014;15(6):e205-e12.

4. Ramachandran A, Snehalatha C, Shetty AS, Nanditha A. Trends in prevalence of diabetes in Asian countries. World journal of diabetes. 2012;3(6):110.

5. Rosella LC, Mustard CA, Stukel TA, Corey P, Hux J, Roos L, et al. The role of ethnicity in predicting diabetes risk at the population level. Ethnicity \& health. 2012;17(4):419-37.

6. Kakar Z, Siddiqui M, Amin R. Prevalence and risk factors of diabetes in adult population of South Asia. Clinical Medicine and Diagnostics. 2013;3(2):18-28.

7. WHO. Cardiovascular diseases (CVDs): Fact sheets 2017 [27th May, 2019]. Available from: https://www.who.int/en/news-room/fact-sheets/detail/cardiovascular-diseases-(cvds). 
8. World Life Expectancy. World Health Ranking 2017 [Available from:

https://www.worldlifeexpectancy.com/world-health-rankings.

9. Lozano R, Naghavi M, Foreman K, Lim S, Shibuya K, Aboyans V, et al. Global and regional mortality from 235 causes of death for 20 age groups in 1990 and 2010: a systematic analysis for the Global Burden of Disease Study 2010. The lancet. 2012;380(9859):2095-128.

10. WHO. Chronic obstructive pulmonary disease (COPD): Fact sheets 2017 [cited 2019 27th May]. Available from: https://www.who.int/en/news-room/fact-sheets/detail/chronic-obstructive-pulmonary-disease-(copd).

11. UN. Sustainable Development Goals 2015 [Available from:

https://www.un.org/sustainabledevelopment/sustainable-development-goals/.

12. Siegel KR, Patel SA, Ali MK. Non-communicable diseases in South Asia: contemporary perspectives. British Medical Bulletin. 2014;111(1):31-44.

13. Kruk ME, Gage AD, Arsenault C, Jordan K, Leslie HH, Roder-DeWan S, et al. High-quality health systems in the Sustainable Development Goals era: time for a revolution. The Lancet Global Health. 2018;6(11):e1196-e252.

14. Bengoa R, Kawar R, Key P, Leatherman S, Massoud R, Saturno P. Quality of care: A process for making strategic choices in health systems. Geneva: World Health Organization. WHO press; 2006.

15. Brocklehurst P, Kujan O, O'Malley LA, Ogden G, Shepherd S, Glenny AM. Screening programmes for the early detection and prevention of oral cancer. Cochrane database of systematic reviews. 2013(11).

16. Hanlon P, Yeoman L, Gibson L, Esiovwa R, Williamson AE, Mair FS, et al. A systematic review of interventions by healthcare professionals to improve management of non-communicable diseases and communicable diseases requiring long-term care in adults who are homeless. BMJ open. 2018;8(4):e020161.

17. Bleich SN, Koehlmoos TL, Rashid M, Peters DH, Anderson G. Noncommunicable chronic disease in Bangladesh: overview of existing programs and priorities going forward. Health policy. 2011;100(2-3):282-9.

18. Tricco AC, Langlois E, Straus SE. Rapid reviews to strengthen health policy and systems: a practical guide: World Health Organization; 2017.

19. Popay J, Roberts H, Sowden A, Petticrew M, Arai L, Rodgers M, et al. Guidance on the conduct of narrative synthesis in systematic reviews. A product from the ESRC methods programme Version. 2006;1:b92.

20. Snilstveit B, Oliver S, Vojtkova M. Narrative approaches to systematic review and synthesis of evidence for international development policy and practice. Journal of development effectiveness. 2012;4(3):409-29.

21. Ali MK, Singh K, Kondal D, Devarajan R, Patel SA, Shivashankar R, et al. Effectiveness of a multicomponent quality improvement strategy to improve achievement of diabetes care goals: a randomized, controlled trial. Annals of internal medicine. 2016;165(6):399-408.

22. Vithana PVSC, Ariyaratne M, Jayawardana P. Quality of breast cancer early detection services conducted by Well Woman Clinics in the district of Gampaha, Sri Lanka. Asian Pacific Journal of Cancer Prevention. 2013;14(1):7580.

23. Ghoshal A, Damani A, Muckaden M, Yennurajalingam S, Salins N, Deodhar J. Patient's Decisional Control Preferences of a Cohort of Patients With Advanced Cancer Receiving Palliative Care in India. Journal of palliative care. 2019;34(3):175-80.

24. Lewis, Newell. Patients' perspectives of care for type 2 diabetes in Bangladesh-a qualitative study. BMC public health. 2014;14(1):737.

25. Mahapatra S, Nayak S, Pati S. Quality of care in cancer: An exploration of patient perspectives. Journal of family medicine and primary care. 2016;5(2):338.

Page 25/28 
26. Mathew A, Agarwal J, Munshi A, Laskar S, Pramesh C, Karimundackal G, et al. A prospective study of telephonic contact and subsequent physical follow-up of radically treated lung cancer patients. Indian journal of cancer. 2017;54(1):241-52.

27. Nayak, Pradhan JPB, Reddy S, Palmer JL, Zhang T, Bruera E. Cancer patients' perception of the quality of communication before and after the implementation of a communication strategy in a regional cancer center in India. Journal of clinical oncology. 2005;23(21):4771-5.

28. Shams S, Jabbar AA, Nanji K, Jan R, Tharani A. Influence of supportive care on chemotherapy patients' self-care behaviour and satisfaction: A pilot study conducted in Karachi, Pakistan. Indian journal of cancer. 2018;55(1):115-21.

29. Tovey P, Broom A, Chatwin J, Hafeez M, Ahmad S. Patient assessment of effectiveness and satisfaction with traditional medicine, globalized complementary and alternative medicines, and allopathic medicines for cancer in Pakistan. Integrative Cancer Therapies. 2005;4(3):242-8.

30. Upadhyay DK, Ibrahim MIM, Mishra P, Alurkar VM. A non-clinical randomised controlled trial to assess the impact of pharmaceutical care intervention on satisfaction level of newly diagnosed diabetes mellitus patients in a tertiary care teaching hospital in Nepal. BMC health services research. 2015;15(1):57.

31. Basu P, Ghoshal M, Chattopadhyay K, Mittal S, Das P, Choudhury D, et al. Cervical screening by visual inspection with acetic acid (VIA) is well accepted by women-results from a community-based study in rural India. Asian Pac J Cancer Prev. 2006;7(4):604-8.

32. Sankaranarayanan R, Ramadas K, Thara S, Muwonge R, Thomas G, Anju G, et al. Long term effect of visual screening on oral cancer incidence and mortality in a randomized trial in Kerala, India. Oral oncology. 2013;49(4):314-21.

33. Singh M, Agarwal A, Sinha V, Manoj Kumar R, Jaiswal N, Jindal I, et al. Application of Handheld Tele-ECG for Health Care Delivery in Rural India. International Journal of Telemedicine and Applications. 2014;2014:1-6.

34. Ghoshal A, Damani A, Muckaden M, Yennurajalingam S, Salins N, Deodhar J. Patient's Decisional Control Preferences of a Cohort of Patients With Advanced Cancer Receiving Palliative Care in India. Journal of palliative care. 2019;34 (3):175-80.

35. Wood R, Viljoen V, Van Der Merwe L, Mash R. Quality of care for patients with non-communicable diseases in the Dedza District, Malawi. African journal of primary health care \& family medicine. 2015;7(1):1-8.

36. Kösters JP, Gøtzsche PC. Regular self-examination or clinical examination for early detection of breast cancer. Cochrane Database of Systematic Reviews. 2003(2).

37. Kane J, Landes M, Carroll C, Nolen A, Sodhi S. A systematic review of primary care models for noncommunicable disease interventions in Sub-Saharan Africa. BMC family practice. 2017;18(1):46.

38. Siddharthan T, Nassali F, Kalyesubula R, Coca S, Rastegar A, Rabin T, et al. An educational booklet for patientcentred health education about a non-communicable disease in low-income and middle-income countries. The Lancet Global Health. 2016;4:S25.

39. Tessema GA, Gomersall JS, Mahmood MA, Laurence CO. Factors determining quality of care in family planning services in Africa: a systematic review of mixed evidence. PLoS One. 2016;11(11):e0165627.

40. Barbazza E, Yegeubayeva S, Akkazieva B, Tsoyi E, Zheleznyakov E, Tello JE. Improving clinical practice in primary care for the prevention and control of noncommunicable diseases: a multi-actor approach to two regional pilot projects in Kazakhstan. Cardiovascular diagnosis and therapy. 2019;9(2):129.

\section{Figures}

Page 26/28 




Figure 1

Flow chart (PRISMA diagram) of the study selection process 


\title{
Risk of Bias of RCT
}

\author{
Other bias \\ Attrition bias \\ Detection bias \\ Performance bias \\ Reporting bias
}

Allocation concealment

Random sequence generation

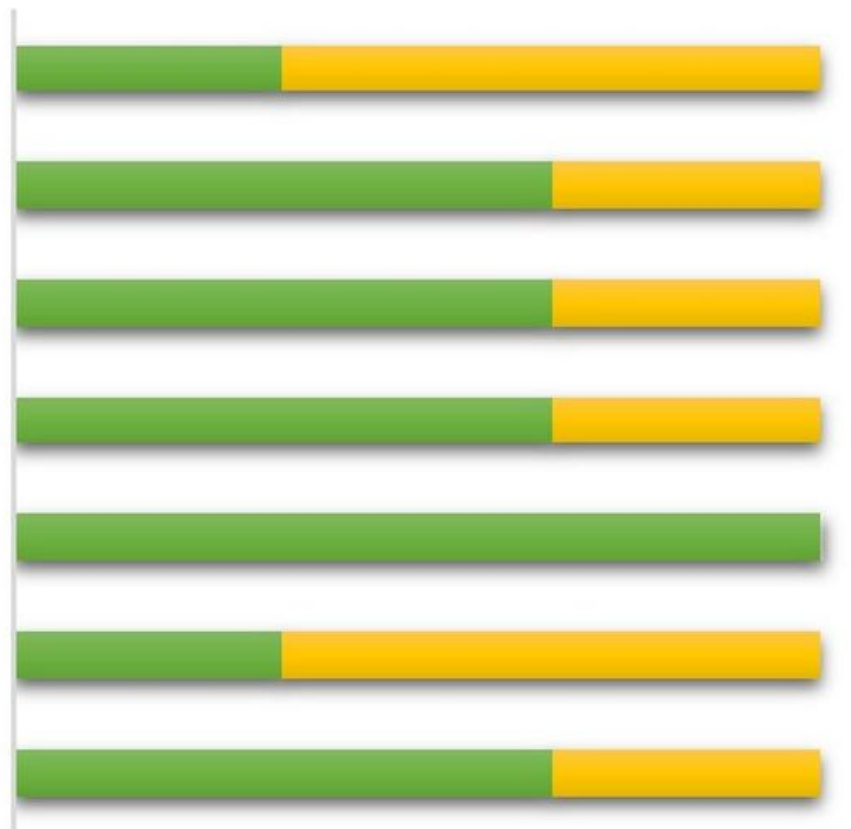

Low risk of bias High risk of bias Unclear risk of bias

Figure 2

Risk of bias of Randomized Controlled Trials

\section{Supplementary Files}

This is a list of supplementary files associated with this preprint. Click to download.

- Supplementaryfile.doc 